\section{RPGRIP1L, FTO and obesity}

$R P G R I P 1 L$ is one of several genes located in the obesityassociated $F T O$ region, and the risk-associated SNP in intron 1 of $F T O$ has been proposed to influence RPGRIP1L expression. To further evaluate a potential role for RPGRIP1L in obesity-related traits, George Stratigopoulos, Rudolph Leibel and colleagues (Cell Metab. 19, 767-779, 2014) examined the phenotype of mice heterozygous for a null allele of Rpgrip1l. They found that these mice exhibited increased weight gain and markedly increased adiposity, accompanied by increased energy intake and increased serum leptin levels. Because RPGRIP1L has previously been implicated in regulating trafficking of the leptin receptor (Lepr) to cilia, the authors examined the hypothalamus in Rpgrip1I heterozygous mice and observed reduced levels of Lepr ciliary localization and of downstream markers of leptin signaling. They also observed increased expression of orexigenic genes encoding Neuropeptide Y (Npy) and Agouti-related protein (Agrp) in the hypothalamus of Rpgrip1/ heterozygous mice compared to wildtype controls. These findings support the hypothesis that the effects of the obesity-associated SNP in the FTO region might be mediated, in part, through altered RPGRIP1L expression and downstream effects on leptin signaling.

\section{RGS2 rescues LRRK2 pathogenicity}

Mutations in LRRK2 are associated with risk of Parkinson's disease. James Collins, Benjamin Wolozin and colleagues have now used a systems biology approach to find new regulators of LRRK2 (Hum. Mol. Genet. doi:10.1093/hmg/ddu202, 2 May 2014). They integrated data from 119 publicly available microarray experiments using RNA from the brain and blood of individuals with Parkinson's disease and controls to build a coexpression network centered on LRRK2 that confirmed known interactions and predicted many new regulators. To validate these predictions, the authors targeted 181 Caenorhabditis elegans genes and 200 human genes (corresponding to 506 putative C. elegans orthologs) exhibiting LRRK2-coordinated expression using RNA interference (RNAi) in worms expressing human LRRK2. They found that $40 \%$ of the genes affected neurite shortening and dopaminergic neuron toxicity, phenotypes associated with $L R R K 2$-driven Parkinson's disease. They further identified RGS2 as a regulatory hub in the network and confirmed that RGS2 physically interacted with LRRK2 in vitro and in vivo. They also found that RGS2 interacted synergistically with LRRK2 to inhibit its kinase activity. Finally, they found that Parkinson's disease cases with the common LRRK2 Gly2019Ser alteration, as well as sporadic cases, had lower levels of RGS2 protein in caudate striatal tissue compared to controls.

\section{iPSC models of ALS}

Dominant mutations in SOD1 (encoding superoxide dismutase 1) cause amyotrophic lateral sclerosis (ALS) and its hallmark feature, motor neuron death. Now, Su-Chun Zhang and colleagues (Cell Stem Cell doi:10.1016/j.stem.2014.03.004, 3 April 2014) and Kevin Eggan and colleagues (Cell Stem Cell doi:10.1016/j.stem.2014.02.004, 3 April 2014) have independently generated induced pluripotent stem cells (iPSCs) from individuals with SOD1 mutations and investigated early pathological events in motor neurons derived from these iPSCs. Both groups

Written by Orli Bahcall, Brooke LaFlamme, Emily Niemitz \& Kyle Vogan created isogenic controls through TALEN-mediated genetic correction of the SOD1 mutation. Zhang and colleagues found that SOD1-mutant motor neurons exhibited neurofilament aggregation and altered stoichiometry for the neurofilament subunits. They showed that mutant SOD1 binds to the 3' UTR of the NFL gene and decreases the amount of NFL mRNA produced. Eggan and colleagues characterized transcriptome changes in SOD1-mutant motor neurons and showed that these cells expressed markers of an unfolded protein response and endoplasmic reticulum (ER) stress. They showed that some of these transcriptional changes are also present in motor neurons derived from the iPSCs of individuals with ALS caused by pathogenic repeat expansions in the C9orf72 locus, suggesting that these distinct disease-causing mutations act through common pathways.

EN

\section{Immune cell-specific eQTLs}

Christophe Benoist, Barbara Stranger, Philip De Jager and colleagues conducted expression quantitative trait locus (eQTL) profiling of CD4 ${ }^{+}$ $\mathrm{T}$ cells and monocytes purified from the blood cells of 461 healthy volunteers from a multi-ancestry cohort (Science 344, 519-523, 2014). They identified cis-eQTLs in each cell type in individuals of African, European and East Asian ancestry within this cohort, finding that over $90 \%$ of these loci were shared across ancestry groups. They identified cell type-specific cis-eQTLs, including 39\% specific to monocytes, $8 \%$ specific to T cells and $62 \%$ shared by both cell types. They also identified 482 trans-eQTL associations involving 55 genes specific to monocytes, 31 specific to T cells and 4 shared by both cell types. They examined the overlap of eQTLs with SNPs associated with disease in the US National Institutes of Health (NIH) catalog of published genome-wide association studies (GWAS) and used a regulatory trait concordance score to identify those suggested to tag the same functional variant. They found an enrichment of cis-eQTLs in SNPs associated with autoimmune disorders. Some diseases showed marked cell type specificity: SNPs associated with multiple sclerosis, rheumatoid arthritis or type 1 diabetes were enriched for T cell-specific cis-eQTLs, whereas SNPs associated with Alzheimer's disease, Parkinson's disease or type 2 diabetes were enriched for monocyte-specific cis-eQTLs.

$O B$

\section{Polar bear genomics}

Rasmus Nielsen, Eske Willerslev, Jun Wang and colleagues have performed a population genomics study of polar bears to gain insights into their evolutionary history and the genetic mechanisms underlying their adaptation to the Arctic environment (Cell 157, 785-794, 2014). The authors generated a de novo assembly of the polar bear genome through deep sequencing of a single polar bear and obtained population diversity data by performing lower-depth sequencing of 79 polar bears and 10 brown bears. From these data, they estimate that polar bears diverged from brown bears roughly 400,000 years ago, with continuous gene flow from polar bears into North American brown bears since their divergence. They also looked for genomic signatures of adaptation and found evidence that genes associated with cardiovascular function and adipose tissue development have evolved under strong positive selection in the polar bear population. The strongest signature of positive selection was found at $A P O B$, which encodes a protein important for the transport of lipids in blood and their uptake by cells. They also found signatures of positive selection at two pigmentation genes that might underlie the phenotype of white coat color in polar bears. 